\title{
UMA DIMENSÃo CULTURAL DA PAISAGEM: HISTÓRIA AMBIENTAL E OS ASPECTOS BIOGEOGRÁFICOS DE UM TABU
}

Rita de Cássia de Paula Freitas Svorc*

Rogério Ribeiro de Oliveira**

Resumo: Na estrutura e composição da Mata Atlântica, especialmente em áreas de florestas secundárias é notável a presença de árvores de grande porte do gênero Ficus (Moraceae), preservados da derrubada por razões culturais por populações tradicionais. A estrutura de trechos de florestas secundárias localizadas no sul do Estado do Rio de Janeiro foi determinada nas proximidades de grandes figueiras por meio de parcelas de $20 \times 5 \mathrm{~m}$. Em três áreas foi amostrado um total de 105 espécies de árvores, sendo que as figueiras atingiram o maior valor de cobertura, sendo responsáveis em média por $43,4 \%$ da área basal. A presença destes exemplares pode ser atribuída a um mesmo tabu cultural, espalhado por extensas regiões do país e que impõem alterações na paisagem florestal.

Palavras-chave: paisagem cultural - biogeografia - Ficus - populações tradicionais florestas secundárias

\section{A CULTURAL LANDSCAPE DIMENSION: ENVIRONMENTAL HISTORY AND BIOGEOGRAPHIC ASPECTS OF A TABOO}

\begin{abstract}
Structure and composition of many areas of secondary Atlantic Forest are marked by the notable presence of large trees of the genus Ficus (Moraceae) that have been spared from felling for cultural reasons when forest areas cleared for farming by traditional populations. Secondary forests structure at South of Rio de Janeiro State located near centenary Ficus trees were examined using $25 \times 5 \mathrm{~m}$ sample plots. A total of 105 tree species were identified in the three areas, and the fig trees had the highest cover values of any species, being responsible for an average of $43.4 \%$ of the basal area. The presence of these large trees can be attributed to the same cultural taboo found in extensive regions of Brazil that imposes landscape transformation.
\end{abstract}

Key Words: cultural landscape - biogeography - Ficus - traditional populations - secondary forests

\section{Introdução: as figueiras e a paisagem florestal}

As paisagens são impregnadas de passado. Dentre muitas outras características, constituem "heranças das sucessivas relações entre homem e natureza" (SANTOS, 2006), podendo ser vistas tanto como um produto da coevolução das sociedades humanas e do meio natural (NAVEH, 2005), quanto à manifestação espacial da relação homemambiente (CRUMLEY, 1994). Assim, em uma perspectiva histórica, é evidente que o legado ambiental que chegou até os dias de hoje é um produto das relações de populações passadas com o meio (GARCÍA-MONTIEL, 2002). Muito do que entendemos hoje por natureza "primitiva" constitui na verdade um mosaico

\footnotetext{
* Email: ritasvorc@gmail.com

** Departamento de Geografia, Centro de Ciências Sociais, Pontifícia Universidade Católica do Rio de Janeiro. Email: rro@puc-rio.br
} 
vegetacional de usos pretéritos ligados à subsistência de populações passadas. Mediante a apropriação da natureza, da sua incorporação na reprodução material e simbólica dos grupos sociais, geram-se marcas na terra. Estes usos e marcas se sobrepõem no espaço com maior ou menor intensidade e deixam vestígios que evidenciam os paleoterritórios, ou seja, a espacialização das resultantes ecológicas decorrentes do uso dos ecossistemas por populações passadas na busca de suas condições de existência (OLIVEIRA, 2008).

A este propósito, a perspectiva da Geografia contribui para aumentar a compreensão da dinâmica da paisagem e fornecer um quadro de referência para avaliar os atuais padrões e processos ecológicos. Segundo Acot (1990), a Geografia vem se mostrando como uma ciência capaz de estabelecer esta relação. No caso da Floresta Atlântica, cuja ocupação humana data de milhares de anos, o longo histórico de transformação de suas condições ambientais é resultante da forma com que suas populações (sejam pré-históricas, de índios, brancos ou negros) interagiram ou interagem com o ambiente. Muitos trabalhos vêm demonstrando que florestas tidas como primárias podem ter sido intensamente manejadas pelo homem no passado, direta ou indiretamente (SIMMONS, 1996; ADAMS, 2000). No Sudeste Brasileiro, extensas áreas utilizadas preteritamente para cultivos de subsistência, geraram florestas secundárias em diversos estágios de regeneração, alterando consideravelmente a composição e a estrutura originais das comunidades (OLIVEIRA, 2002).
No presente estudo, tem-se como ponto de partida a análise biogeográfica da estrutura e composição de alguns destes trechos de florestas secundárias, onde é conspícua a presença de exemplares de figueiras de grande porte, algumas com diâmetro superior a 2,5 m. Embora no Brasil existam cerca de 100 espécies nativas (CARAUTA, 1989), o gênero Ficus se destaca, além do aspecto alimentar

(representado fundamentalmente por Ficus carica L., o figo comestível), por uma histórica tradição cultural por quase todo o mundo. $\mathrm{Na}$ costa da África, o iroko é considerado uma árvore sagrada pelos praticantes de candomblé (Verger 1995). De acordo com Fonseca (2005), na tradição afro descendente brasileira - por sua vez uma confluência de pelo menos quatro tradições ancestrais africanas - as figueiras ocupam o lugar da espécie africana (Clorophora excelsa (Welw.) Benth. para representar um deus-árvore: o iroko. Segundo Carauta e Diaz (2003), um fato interessante ocorreu com a vinda dos rituais religiosos do candomblé do continente africano para o Brasil. Aqui, algumas figueiras nativas (como Ficus glabra Vell., $F$. gomelleira Kunth., $F$. cyclophylla (Miq.) Miq. ou ainda $F$. adhantifolia Schott) substituem a morácea africana nos ritos do candomblé, mas a designação iroko permaneceu a mesma, sendo suas folhas utilizadas em rituais de iniciação.

No Antigo Testamento da Bíblia existem perto de 40 referências às figueiras, sendo que no Novo Testamento podem ser encontradas 16 citações. No evangelho de S. Mateus (21: 18-22), Jesus seca (e não amaldiçoa, como se vê 
na tradição popular) uma figueira que não dá fruto. Assim, seja pela tradição judaico-cristã ou pela afro-brasileira, esta árvore é portadora de forte simbolismo e, possivelmente por estes motivos, é geralmente preservada do corte por populações interioranas quando promovem derrubadas para a implantação de suas roças. Este mesmo tabu foi constatado com moradores mais antigos nas áreas onde foi realizado o presente trabalho.

São comportamentos que, ao longo do tempo, vão deixando na paisagem marcas tanto materiais como imateriais, assumindo formas diversas. Dentre elas destacam-se as alterações na composição, estrutura e funcionalidade dos ecossistemas e que podem ser detectadas com o uso de ferramentas apropriadas da Biogeografia. No entanto, no caso dos aspectos não materiais da paisagem, como a sua dimensão simbólica, a sua constatação torna-se mais difícil. Neste caso, a História Ambiental podem trazer importantes subsídios para a compreensão das resultantes ecológicas da transformação da paisagem. Além disso, segundo Camargo, (1998 apud SOUZA e MARIANO (2008, p. 89)), cabe à Biogeografia, mais do que a explicação acerca da distribuição dos seres vivos, a conjunção dos estudos dos processos biológicos, juntamente com os derivados da Sociedade, representando assim um paradigma para a interpretação dos padrões e mudanças nas paisagens naturais e culturais da atualidade, bem como das paisagens passadas. Neste contexto, o presente trabalho tem como objetivo central avaliar a presença de um marco na paisagem (as figueiras centenárias), relacionando-as à estrutura e composição da Mata Atlântica remanescente de usos pretéritos.

\section{Procedimentos metodológicos}

O trabalho foi desenvolvido em duas Unidades de Conservação localizadas no Sul do Estado do Rio de Janeiro, no município de Angra dos Reis: o Parque Nacional da Serra da Bocaina e o Parque Estadual da Ilha Grande. Estas se encontram dentro de um mesmo território de uso da terra por roças de subsistência de sitiantes e caiçaras e, por esta razão, a grande maioria dos seus remanescentes florestais são secundários. As amostragens realizadas no perímetro do Parque Nacional da Serra da Bocaina, na bacia hidrográfica do Rio Mambucaba, localizaram-se em altitude aproximada de 300 m. s. m., portanto no domínio da Floresta Ombrófila Densa submontana. Segundo Guimarães et al. (2000), o clima geral pode ser definido como temperado superúmido (tU), com médias anuais de temperatura abaixo de $16^{\circ} \mathrm{C}$ e precipitações pluviométricas em torno de $1.800 \mathrm{~mm}$. A área de trabalho do Parque Estadual da Ilha Grande, também localizada no município de Angra dos Reis, localizou-se a 200 m.s.m. O clima da região é quente-úmido, com temperatura média anual de $23^{\circ} \mathrm{C}$ e pluviosidade anual de aproximadamente $2.200 \mathrm{~mm}$. Tanto na Bocaina quanto na Ilha Grande, as formações secundárias - geralmente em estágio avançado de regeneração ocupam a grande maioria de suas encostas e são reconhecidas por espécies 
indicadoras como o jacatirão (Miconia cinnamomifolia (DC.) Naud.), o guapuruvú (Schizolobium parahyba (Vell.) Blake) e outras (DELAMONICA et al., 2002).

Para fins de análise, consideram-se como figueiras centenárias os exemplares de grande porte, pelo menos no que se refere ao diâmetro do caule, pertencentes ao gênero Ficus (geralmente Ficus ciclophylla (Miq.) Miq., F. clusiifolia Schott, F. gomelleira Kunth., F. insipida Willd., F. pulchela Scott e F. glabra Vell.) e que muito comumente são encontradas na Mata Atlântica em formações secundárias na faixa de 30-150 anos de regeneração (Fig. $1)$.

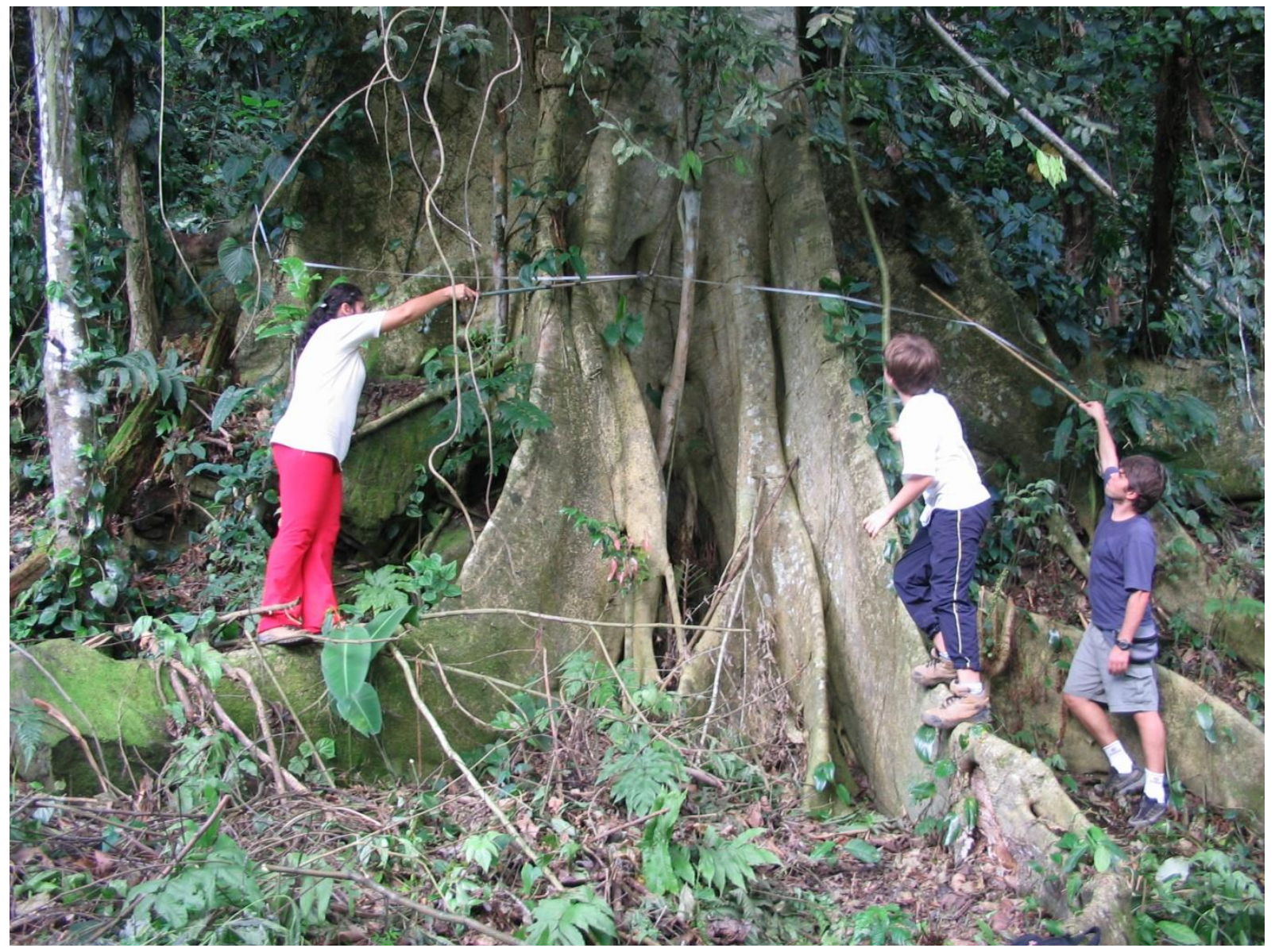

Exemplar de Ficus glabra Vell. em trato de floresta secundária no Parque Nacional da Bocaina.

Foram estabelecidas três áreas amostrais demarcadas pela presença de figueiras centenárias, a saber: duas na Serra da Bocaina (Bocaina I e II) e uma na Ilha Grande. Em todas as foram alocadas 10 parcelas de $20 \times 5 \mathrm{~m}$, resultando um total de $1.000 \mathrm{~m}^{2}$ por área. Cada unidade de estudo foi constituída de uma faixa de $100 \mathrm{~m}$ de extensão em duas direções (opostas), tendo como ponto central uma figueira centenária, que serviu como referência central para o estabelecimento das parcelas (Fig. 2). A forma retangular das mesmas foi escolhida para permitir comparações estruturais relativas à proximidade ou distância da figueira de referência. O critério de inclusão foram árvores e arbustos com o diâmetro à altura do peito (dap) $\geq 5 \mathrm{~cm}$. Todas as áreas amostradas tratavam-se, segundo relato de

* Email: ritasvorc@gmail.com

** Departamento de Geografia, Centro de Ciências Sociais, Pontifícia Universidade Católica do Rio de Janeiro. Email: rro@puc-rio.br 
moradores antigos, de antigas roças com idades na faixa de 40-80 anos de regeneração. Nas redondezas foram encontrados vestígios que confirmavam este uso pretérito, como presença de carvão no solo, de espécies frutíferas cultivadas, ruínas de baldrames de casas ou de antigos fogões.

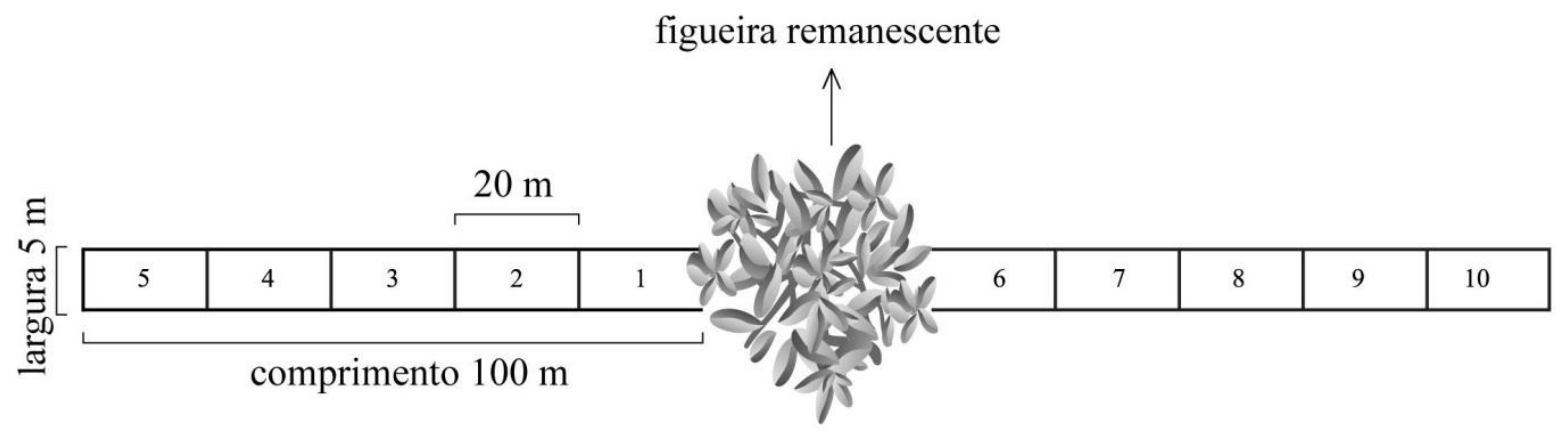

Figura 2. Disposição das parcelas nas áreas de estudos em relação à figueira utilizada como referência.

Os parâmetros fitossociológicos foram Densidade e Dominância absolutas e relativas e, a partir destes, o valor Cobertura (IVC), segundo Vuono (2002):

a) densidade total por área (DTA): representa o número total de indivíduos de todas as espécies por unidade de área.

\section{DTA $=\mathbf{N} /$ 1ha}

onde: $\mathrm{N}=$ número total de indivíduos amostrados

b) densidade específica relativa $\left(D R_{s}\right)$ : representa o número médio de árvores de uma determinada espécie (s) por unidade de área.

$$
D R_{s}=\left(n_{s} / N\right) .100
$$

onde: $\mathrm{n}_{\mathrm{s}}=$ número de indivíduos amostrados da espécie $\mathrm{s} ; \mathrm{N}=$ número total de indivíduos amostrados.

c) área basal individual: representa a área ocupada pelo tronco de cada indivíduo.

$$
A B I_{s}=D^{2} n / 4
$$

onde: $\mathrm{D}$ = diâmetro de cada indivíduo da espécie s. 
d) área basal por por espécie $\left(A B_{s}\right)$ : representa a média das áreas basais dos indivíduos de uma espécie.

$$
A B_{s}=\Sigma A B I_{s} / n_{s}
$$

onde: $\mathrm{n}_{\mathrm{s}}=$ número de indivíduos amostrados da espécie $\mathrm{s}$.

e) dominância relativa por espécie: representa a relação percentual entre a área basal total de uma espécie e a área basal total de todas as espécies amostradas.

\section{$\operatorname{DoR}_{s}=\left(\Sigma \mathrm{ABI}_{s} / \mathrm{ABT}\right) \cdot 100$}

onde: $\mathrm{ABI}_{\mathrm{s}}=$ área basal de cada indivíduo da espécie $\mathrm{s} ; \mathrm{ABT}=$ soma das áreas basais de todas as espécies amostradas.

f) valor de cobertura: representa o grau em que a espécie se encontra bem estabelecida na comunidade e resulta dos valores relativos de densidade e dominância, atingindo, portanto o valor máximo de 200 .

$$
\mathrm{VC}=\mathrm{DR}_{\mathrm{s}}+\mathrm{Do}_{\mathrm{s}}
$$

Após secagem em estufa, as amostras foram identificadas utilizando-se a coleção do Herbário do Departamento de Botânica da UFRRJ (onde se encontra depositado materialtestemunho) e com consultas a especialistas. A sinonímia e a grafia dos taxa foram atualizadas mediante consulta ao índice de espécies do banco de dados do Missouri Botanical Garden, disponível na página http://mobot.mobot.org/W3T/Search/vast.html, sendo que o sistema de classificação adotado foi o APG II (2003). Foram também realizadas coletas de amostras de solo nas profundidades de 0-10 cm nas áreas de estudos. Em cada área foram tomadas três amostras compostas, formadas por dez amostras simples. As análises foram feitas no Departamento de Solos da Universidade Federal Rural do Rio de Janeiro (UFRRJ) e os dados foram comparados entre si com o uso do teste de Tukey (ZAR, 1996).

\section{. Resultados e discussão}

Nos pontos da Mata Atlântica estudados foram encontrados vários exemplares de figueiras com porte significativamente superior ao dos demais componentes arbóreos. Estas figueiras são frequentes em formações secundárias do Sudeste Brasileiro e, em função disso, integram a toponímia de numerosos locais (ex: Mata da Figueira, Grota da Gameleira, etc.). Mesmo em pastagens ou em áreas urbanizadas de diversas capitais brasileiras, frequentemente as árvores de maior porte são figueiras centenárias.

A Tabela 1 apresenta as características químicas dos solos das áreas de estudos. À exceção do fósforo, todos os demais parâmetros nas três áreas estudadas não diferem entre si pelo teste de Tukey a 5\%. Em relação à acidez, medida pelo pH, observa-se que os solos 
das áreas Bocaina II e Ilha Grande se mostraram moderadamente ácidos e a área Bocaina I apresentou o solo mais próximo à neutralidade. O maior teor de alumínio encontrado na área Bocaina II pode estar relacionado ao seu menor índice de $\mathrm{pH}(5,4)$, uma vez que a precipitação de sais e hidróxidos de alumínio ocorre em $\mathrm{pH}$ mais elevado, a partir do $\mathrm{pH}$ 5,4 (Sollins 1998). Destaque maior deve ser dado para os baixos valores de fósforo, ainda que a área Bocaina I tenha apresentado um resultado de $\mathrm{P}$ superior aos demais. Apesar do valor V\% não apresentar diferenças entre as áreas, nesta última este parâmetro apresentou um valor superior a $70 \%$.

Tabela 1. Características químicas dos solos das três áreas de estudos. Médias seguidas por letras distintas, na mesma coluna, diferem entre si pelo teste de Tukey a $5 \%$.

\begin{tabular}{|c|c|c|c|c|c|c|c|c|c|c|c|c|}
\hline \multirow{2}{*}{ Área } & $\mathrm{Na}$ & $\mathrm{Ca}$ & $\mathrm{Mg}$ & K & $\mathrm{H}+\mathrm{Al}$ & Al & S & $\mathrm{T}$ & \multirow{2}{*}{$\begin{array}{l}\mathrm{V} \\
\%\end{array}$} & \multirow{2}{*}{$\begin{array}{l}\mathrm{pH} \\
\text { água } \\
1: 2,5\end{array}$} & $P$ & K \\
\hline & \multicolumn{8}{|c|}{ 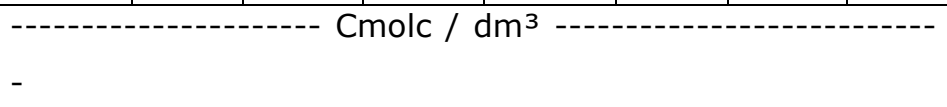 } & & & \multicolumn{2}{|c|}{---- mg/L ---- } \\
\hline $\begin{array}{l}\text { Bocaina } \\
\text { I }\end{array}$ & $0,05^{a}$ & $6,8^{\mathrm{a}}$ & $4,4^{a}$ & $0,7^{\mathrm{a}}$ & $4,4^{a}$ & $0,0^{a}$ & 12,0 & 16,4 & $72,9^{a}$ & $6,0^{\mathrm{a}}$ & $4,7^{\mathrm{a}}$ & $266,0^{a}$ \\
\hline $\begin{array}{l}\text { Bocaina } \\
\text { II }\end{array}$ & $0,04^{a}$ & $4,3^{\mathrm{a}}$ & $3,4^{a}$ & $0,4^{\mathrm{a}}$ & $5,9^{a}$ & $0,8^{a}$ & 8,2 & 14,1 & $54,3^{a}$ & $5,4^{a}$ & $2,7^{b}$ & $167,3^{\mathrm{a}}$ \\
\hline $\begin{array}{l}\text { Ilha } \\
\text { Grande }\end{array}$ & $0,06^{a}$ & $3,8^{a}$ & $1,7^{a}$ & $0,2^{a}$ & $5,5^{a}$ & $0,1^{\mathrm{a}}$ & 5,8 & 11,3 & $50,9^{a}$ & $5,6^{a}$ & $2,0^{b}$ & $93,3^{a}$ \\
\hline
\end{tabular}

Assim, em função das três áreas não diferirem entre si em termos estatísticos no que se refere aos parâmetros analisados (exceto fósforo), do ponto de vista da vegetação podese considerar que o conteúdo químico do solo não representa um fator relevante na diferenciação da mesma, o que valida a comparação da vegetação das três áreas.

Com relação aos principais descritores da vegetação das áreas estudadas, em função do maior tempo sucessional, a área da Ilha Grande apresentou maior riqueza de espécies, assim como maior densidade (Tab. 2). Na escala utilizada, as figueiras foram responsáveis por parte significativa da área basal (50,4\%, 32,9\% e 47,9\%), respectivamente nas áreas Bocaina I, Bocaina II e Ilha Grande. A porcentagem de troncos mortos em pé é compatível com áreas secundárias avançadas (Oliveira 2002).

Tabela 2. Descritores da vegetação das três áreas de estudo, Angra dos Reis, RJ.

\begin{tabular}{|l|c|c|c|}
\hline \multicolumn{1}{|c|}{ Parâmetro } & Bocaina I & Bocaina II & Ilha Grande \\
\hline Riqueza de espécies & 32 & 32 & 63 \\
\hline Troncos mortos em pé & $8,2 \%$ & $3,7 \%$ & $6,4 \%$ \\
\hline Densidade (ind.ha $\left.{ }^{-1}\right)$ & 610 & 1.340 & 1.720 \\
\hline Área basal total $\left(\mathrm{m}^{2} . \mathrm{ha}^{-1}\right)$ & 50,2 & 75,7 & 76,2 \\
\hline
\end{tabular}




\begin{tabular}{|l|l|l|l|}
\hline \begin{tabular}{l} 
Área basal $\begin{array}{l}\left(\mathrm{m}^{2} \cdot \mathrm{ha}^{-1}\right) \\
\text { (excluindo as figueiras) }\end{array}$ \\
\hline
\end{tabular} & 24,9 & 50,8 & 39,7 \\
\hline
\end{tabular}

No inventário das três áreas de estudo foi amostrado um total de 105 espécies, subordinadas a 34 famílias (Tab. 3). Neste total estão incluídas as morfo-espécies, ou seja, as espécies cuja determinação a nível específico não foi possível. Apesar da relativa dessemelhança entre as áreas Bocaina I e II, a riqueza de espécies foi a mesma (32 espécies) e praticamente a metade da observada em Ilha Grande (63 espécies).

Tabela 3. Espécies ocorrentes nas áreas amostradas com indicação do local de ocorrência.

\begin{tabular}{|c|c|c|c|}
\hline família/espécie & Bocaina I & Bocaina II & Ilha Grande \\
\hline \multicolumn{4}{|l|}{ Annonaceae } \\
\hline Anaxagorea dolichocarpa Sprague \& Sandwith & & & $\mathrm{x}$ \\
\hline Guatteria nigrescens Mart. & $\mathrm{x}$ & & \\
\hline Rollinia dolabripetala (Raddi) R. E. Fr. & & & $x$ \\
\hline Xilopia sericea A. ST.-Hil. & & & $\mathrm{x}$ \\
\hline \multicolumn{4}{|l|}{ Apocynaceae } \\
\hline Geissospermum vellossi (Vell.) Miers. & $\mathrm{x}$ & & \\
\hline Tabernaemontana laeta Mart. & & & $\mathrm{x}$ \\
\hline \multicolumn{4}{|l|}{ Araliaceae } \\
\hline Didymopanax longipetiolatum March. & & & $\mathrm{x}$ \\
\hline \multicolumn{4}{|l|}{ Bombacaceae } \\
\hline Eriotheca pentaphylla (Vell.) A. Robyns & & $\mathrm{x}$ & $x$ \\
\hline Chorisia speciosa St. Hil. & & & $\mathrm{x}$ \\
\hline Bombacaceae sp. & & & $\mathrm{x}$ \\
\hline \multicolumn{4}{|l|}{ Boraginaceae } \\
\hline Cordia sp. & & $\mathrm{x}$ & \\
\hline \multicolumn{4}{|l|}{ Caricaceae } \\
\hline Carica papaya L. & $x$ & & \\
\hline Jacaratia spinosa (Aubl.) A. DC. & $\mathrm{x}$ & & $\mathrm{x}$ \\
\hline \multicolumn{4}{|l|}{ Cecropiaceae } \\
\hline Cecropia sp. & & & $x$ \\
\hline Cecropia glaziovi Snethl. & $\mathrm{x}$ & & \\
\hline \multicolumn{4}{|l|}{ Clusiaceae } \\
\hline Garcinia gardneriana (Planch. \& Triana) Zappi & & $x$ & \\
\hline \multicolumn{4}{|l|}{ Elaeocarpaceae } \\
\hline Sloanea monosperma Vell. & & $\mathrm{x}$ & $\mathrm{x}$ \\
\hline \multicolumn{4}{|l|}{ Erytroxylaceae } \\
\hline Erythroxylum cuspidifolium Mart. & & $x$ & $x$ \\
\hline Erythroxylum passerinum Mart. & & & $x$ \\
\hline
\end{tabular}




\begin{tabular}{|c|c|c|c|}
\hline família/espécie & Bocaina I & Bocaina II & Ilha Grande \\
\hline \multicolumn{4}{|l|}{ Euphorbiaceae } \\
\hline Actinostemum comunis (Müll. Arg.) Pax & & & $x$ \\
\hline Actinostemum sp. & $\mathrm{x}$ & & \\
\hline Aparisthmium cordatum (A. Juss.) Baill. & & & $x$ \\
\hline Euphorbiaceae sp.1 & & & $\mathrm{x}$ \\
\hline Euphorbiaceae sp.2 & & & $\mathrm{x}$ \\
\hline Euphorbiaceae sp.3 & & & $x$ \\
\hline Euphorbiaceae sp.4 & $\mathrm{x}$ & & \\
\hline Hieronyma alchorneoides Allemão & & $\mathrm{x}$ & \\
\hline Maprounea guianensis Aublet & & & $\mathrm{x}$ \\
\hline Pachystroma longifolium (Nees.) Johnston. & & $\mathrm{x}$ & \\
\hline Pausandra sp. & & & $x$ \\
\hline Pausandra trianae (Müll. Arg.) Baill. & & & $\mathrm{x}$ \\
\hline Pera glabrata (Schott) Baill. & & & $x$ \\
\hline Senefeldera multiflora Mart. & & $x$ & $x$ \\
\hline \multicolumn{4}{|l|}{ Hipocrateaceae } \\
\hline Salacia sp. & & $x$ & \\
\hline \multicolumn{4}{|l|}{ Lauraceae } \\
\hline Cryptocarya saligna Mez. & & $x$ & \\
\hline Cryptocarya riedeliana (P. L. R. Moraes) & $\mathrm{x}$ & & \\
\hline Endlicheria glomerata Mez. & & & $x$ \\
\hline Nectandra membranacea (Sw.) Griseb. & $x$ & $x$ & $\mathrm{x}$ \\
\hline Ocotea glaziovi Mez & $\mathrm{x}$ & & \\
\hline \multicolumn{4}{|l|}{ Leguminosae } \\
\hline Albizia polycephala (Benth.) Killip. ex. Record & & & $\mathrm{x}$ \\
\hline Inga flagelliformis (Vell.) Martius & & & $\mathrm{x}$ \\
\hline Inga lanceifolia Benth. & & & $\mathrm{x}$ \\
\hline Pseudopiptadenia inaequalis (Benth.) Rauschert & & & $x$ \\
\hline Zygia latifolia (L.) Fawc. \& Rendle & & & $x$ \\
\hline \multicolumn{4}{|l|}{ Malpighiaceae } \\
\hline Bunchosia sp. & $\mathrm{x}$ & & \\
\hline Byrsonima laxiflora Griseb. & & & $\mathrm{x}$ \\
\hline \multicolumn{4}{|l|}{ Melastomataceae } \\
\hline Miconia calvescens DC. & $\mathrm{x}$ & $\mathrm{x}$ & $\mathrm{x}$ \\
\hline Mouriri sp. & & & $x$ \\
\hline \multicolumn{4}{|l|}{ Meliaceae } \\
\hline Cedrela fissilis Vell. & $\mathrm{x}$ & & \\
\hline Guarea guidonia (L.) Sblumes & $\mathrm{x}$ & $\mathrm{x}$ & \\
\hline Guarea macrophyla Vahl. & & & $x$ \\
\hline
\end{tabular}




\begin{tabular}{|c|c|c|c|}
\hline família/espécie & Bocaina I & Bocaina II & Ilha Grande \\
\hline Trichillia elegans A. Juss. & $\mathrm{x}$ & & \\
\hline \multicolumn{4}{|l|}{ Monimiaceae } \\
\hline Mollinedia schottiana (Spreng.) Perk. & & & $x$ \\
\hline \multicolumn{4}{|l|}{ Moraceae } \\
\hline Brosimum discolor Schott. & & $x$ & \\
\hline Brosimum guianense (Aublet) Huber & & $x$ & \\
\hline Brosimum sp. & & $x$ & \\
\hline Ficus cyclophyla (Miq.) Miq. & & & $x$ \\
\hline Ficus glabra Vell. & $\mathrm{x}$ & & \\
\hline Ficus insipida Willd. & $x$ & $x$ & \\
\hline Sorocea guilheminiana Gaudich. & & $x$ & \\
\hline \multicolumn{4}{|l|}{ Myristicaceae } \\
\hline Virola oleifera (Schott) A. C. Smith & $\mathrm{x}$ & $\mathrm{x}$ & \\
\hline Virola gardneri (A. DC.) Warb. & & & $x$ \\
\hline \multicolumn{4}{|l|}{ Myrtaceae } \\
\hline Calyptranthes lucida Mart. ex DC. & & & $x$ \\
\hline Campomanesia guaviroba (DC.) Kiaersk & & $x$ & \\
\hline Eugenia magnibracteolata (Mattos) D. Legrand & & & $x$ \\
\hline Eugenia rotundifolia Casar. & & & $x$ \\
\hline Gomidesia blanchetiana O. Berg & & & $x$ \\
\hline Gomidesia schaueriana O. Berg & & & $\mathrm{x}$ \\
\hline Gomidesia sp. 1 & & & $x$ \\
\hline Marlierea sylvatica (Gardner) Kiaersk. & $\mathrm{x}$ & $x$ & \\
\hline Myrceugenia myrcioides (Cambess) O. Berg. & & & $\mathrm{x}$ \\
\hline Myrcia laxiflora Cambess. & & & $x$ \\
\hline \multicolumn{4}{|l|}{ Nyctaginaceae } \\
\hline Guapira opposita (Vell.) Reitz & & $x$ & $x$ \\
\hline \multicolumn{4}{|l|}{ Palmae } \\
\hline Astrocarium aculeatissimum (Schott) Burret & $x$ & $x$ & \\
\hline Euterpe edulis Mart. & $x$ & $x$ & \\
\hline \multicolumn{4}{|l|}{ Phytolacaceae } \\
\hline Gallesia integrifolia (Spreng.) Harms & $x$ & $x$ & \\
\hline \multicolumn{4}{|l|}{ Piperaceae } \\
\hline Piper rivinoides Kunth & & $x$ & \\
\hline \multicolumn{4}{|l|}{ Rubiaceae } \\
\hline Alseis floribunda Schum. & $x$ & & \\
\hline Amaioua intermedia Mart. & & & $x$ \\
\hline Bathysa mendonçaei Schum. & $x$ & $x$ & \\
\hline Bathysa nicholsonii K. Shum. & & $x$ & $x$ \\
\hline
\end{tabular}




\begin{tabular}{|c|c|c|c|}
\hline família/espécie & Bocaina I & Bocaina II & Ilha Grande \\
\hline Bathysa stipulata Presl. & $\mathrm{x}$ & $\mathrm{x}$ & $\mathrm{x}$ \\
\hline Chomelia estrellana Müll. Arg. & & & $\mathrm{x}$ \\
\hline Coussarea accedens Müll. Arg. & & $\mathrm{x}$ & $\mathrm{x}$ \\
\hline Coussarea nodosa (Benth.) Müll. Arg. & & & $\mathrm{x}$ \\
\hline \multicolumn{4}{|l|}{ Coussarea sp. } \\
\hline Psychotria barbiflora DC. & & & $\mathrm{x}$ \\
\hline Psychotria carthaginensis Jacq. & & & $\mathrm{x}$ \\
\hline Rustia formosa (Cham. \& Schltdl. ex DC.) Klotzsch & & & $\mathrm{x}$ \\
\hline \multicolumn{4}{|l|}{ Rutaceae } \\
\hline Almeidea rubra A. St. Hill. & $x$ & & $\mathrm{x}$ \\
\hline Conchocarpus aff. cuneifolius (A. ST.-Hill.) & & $\mathrm{x}$ & \\
\hline Pilocarpus spicatus St. Hill. & $\mathrm{x}$ & & \\
\hline Rutaceae sp. 1 & & & $\mathrm{x}$ \\
\hline Rutaceae sp. 2 & & & $\mathrm{x}$ \\
\hline \multicolumn{4}{|l|}{ Sapindaceae } \\
\hline Allophylus heterophyllus Radlk. & & & $\mathrm{x}$ \\
\hline \multicolumn{4}{|l|}{ Sapotaceae } \\
\hline Chrysophyllum flexuosum Mart. & & & $\mathrm{x}$ \\
\hline Chrysophyllum gonocarpum (Mart. \& Eichler) Engl. & $x$ & & \\
\hline Chrysophyllum imperiale Bentham & $\mathrm{x}$ & & \\
\hline Eclynusa ramiflora Mart. & & & $x$ \\
\hline Sapotaceae sp. & & & $\mathrm{x}$ \\
\hline \multicolumn{4}{|l|}{ Siparunaceae } \\
\hline Siparuna guianensis Aubl. & & & $\mathrm{x}$ \\
\hline \multicolumn{4}{|l|}{ Solanaceae } \\
\hline Solanaceae sp. & $x$ & & \\
\hline \multicolumn{4}{|l|}{ Ulmaceae } \\
\hline Trema micrantha (L.) Blume & $x$ & & \\
\hline \multicolumn{4}{|l|}{ Urticaceae } \\
\hline Urera baccifera (L.) Gaud.-Boupré. & $x$ & & \\
\hline \multicolumn{4}{|l|}{ Violaceae } \\
\hline Amphirrhox longifolia (St. Hill.) Spreng. & & $x$ & \\
\hline
\end{tabular}

Feita a análise fitossociológica, as tabelas 4, 5 e 6 foram elaboradas com os parâmetros das 10 espécies que se posicionaram com maior valor de cobertura nas três áreas de estudos. 
Tabela 4. Parâmetros fitossociológicos da área Bocaina I, Parque Nacional da Bocaina (N $=\mathrm{n}$. de indivíduos; $\mathrm{DR}=$ densidade relativa; $\mathrm{DoR}=$ dominância relativa; $\mathrm{VC}=$ valor de cobertura).

\begin{tabular}{|l|l|l|l|l|}
\hline Espécie & N & DR & DoR & VC \\
\hline Ficus glabra & 2 & 3,57 & 48,64 & 52,21 \\
\hline Virola oleifera & 9 & 16,07 & 18,64 & 34,71 \\
\hline Cryptocarya riedeliana & 4 & 7,14 & 1,21 & 8,36 \\
\hline Ficus insipida & 2 & 3,57 & 4,16 & 7,73 \\
\hline Jacaratia spinosa & 2 & 3,57 & 3,62 & 7,19 \\
\hline Nectandra membranacea & 3 & 5,36 & 1,80 & 7,16 \\
\hline Trichillia elegans & 3 & 5,36 & 0,29 & 5,65 \\
\hline Guatteria nigrescens & 1 & 1,79 & 3,81 & 5,59 \\
\hline Bathysa mendonçaei & 2 & 3,57 & 1,82 & 5,39 \\
\hline Chrysophyllum imperiale & 1 & 1,79 & 2,87 & 4,65 \\
\hline
\end{tabular}

Tabela 5. Parâmetros fitossociológicos da área Bocaina II, Parque Nacional da Bocaina ( $\mathrm{N}=$ $\mathrm{n}$. de indivíduos; $\mathrm{DR}=$ densidade relativa; $\mathrm{DoR}=$ dominância relativa; $\mathrm{VC}=$ valor de cobertura).

\begin{tabular}{|l|l|l|l|l|}
\hline Espécie & $\mathrm{N}$ & $\mathrm{DR}$ & $\mathrm{DoR}$ & $\mathrm{VC}$ \\
\hline Ficus insipida & 3 & 2,33 & 33,71 & 36,03 \\
\hline Hieronyma alchornioides & 4 & 3,10 & 18,28 & 21,38 \\
\hline Virola oleifera & 20 & 15,50 & 4,56 & 20,06 \\
\hline Bathysa nicholsonii & 8 & 6,20 & 3,96 & 10,17 \\
\hline Bathysa mendonçaei & 3 & 2,33 & 6,91 & 9,24 \\
\hline Astrocarium aculeatissimum & 9 & 6,98 & 1,11 & 8,09 \\
\hline Sorocea guilheminiana & 8 & 6,20 & 1,11 & 7,31 \\
\hline Euterpe edulis & 8 & 6,20 & 0,73 & 6,93 \\
\hline Senefeldera multiflora & 8 & 6,20 & 0,31 & 6,51 \\
\hline Coussarea accedens & 4 & 3,10 & 2,22 & 5,32 \\
\hline
\end{tabular}

Tabela 6. Parâmetros fitossociológicos da área de estudos da Ilha Grande, Parque Estadual da Ilha Grande $(\mathrm{N}=\mathrm{n}$. de indivíduos; $\mathrm{DR}=$ densidade relativa; $\mathrm{DoR}=$ dominância relativa; $\mathrm{VC}=$ valor de cobertura).

\begin{tabular}{|l|l|l|l|l|}
\hline Espécie & N & DR & DoR & VC \\
\hline Ficus cyclophyla & 2 & 1,24 & 48,3820 & 49,62 \\
\hline Mabea fistulifera & 23 & 14,29 & 10,1326 & 24,42 \\
\hline Jacaratia spinosa & 3 & 1,86 & 8,4748 & 10,34 \\
\hline Psychotria carthaginensis & 6 & 3,73 & 1,2599 & 4,99 \\
\hline Maprounea guianensis & 6 & 3,73 & 1,1671 & 4,89 \\
\hline
\end{tabular}




\begin{tabular}{|l|l|l|l|l|}
\hline Coussarea nodosa & 6 & 3,73 & 0,9814 & 4,71 \\
\hline Bathysa nicholsonii & 4 & 2,48 & 2,1618 & 4,65 \\
\hline Nectandra membranacea & 4 & 2,48 & 1,9231 & 4,41 \\
\hline Aparisthmium cordatum & 4 & 2,48 & 1,7241 & 4,21 \\
\hline Anaxagorea dolichocarpa & 4 & 2,48 & 1,2467 & 3,73 \\
\hline
\end{tabular}

As primeiras dez espécies em valor de cobertura contribuíram com um percentual expressivo desta variável. Os valores de dominância relativa destas 10 espécies nas três áreas de estudo estão acima de $70 \%$ do total máximo que esta variável pode assumir, que é 200. Em florestas tropicais a maioria das espécies ocorre em baixa densidade, não sendo incomum que 5 a 10 espécies representem $50 \%$ do valor de cobertura (HARTSHORN, 1980). Por este motivo, o percentual do valor de cobertura das primeiras dez espécies, foi exibido incluindo os Ficus e excluindo-os (Tab. 7).

Tabela 7. Percentual das primeiras dez espécies com maiores valores de cobertura encontradas nas áreas de estudo.

\begin{tabular}{|l|l|l|l|}
\hline \multicolumn{1}{|c|}{ Característica } & Bocaina I & Bocaina II & Ilha Grande \\
\hline Densidade relativa & $52 \%$ & $58 \%$ & $38,5 \%$ \\
\hline Dominância relativa & $87 \%$ & $72,9 \%$ & $77,4 \%$ \\
\hline Valor de Cobertura com Ficus* & $69,3 \%$ & $65,5 \%$ & $58 \%$ \\
\hline Valor de Cobertura sem Ficus* & $43,2 \%$ & $47,5 \%$ & $33,2 \%$ \\
\hline
\end{tabular}

* = Valores expressos em porcentagem de participação no valor máximo de cobertura total

Pode-se observar uma queda significativa nos valores de cobertura das dez primeiras espécies quando se desconsidera o valor de cobertura de Ficus spp. Houve uma redução de $26,1 \%, 18,0 \%$ e $24,8 \%$ dos valores de cobertura, respectivamente em Bocaina I, Bocaina II e Ilha Grande. Apesar da baixa densidade observada para as espécies do gênero Ficus observada nas três áreas, as mesmas foram responsáveis pelos elevados valores de cobertura obtidos por se tratar de árvores com diâmetros elevados. Na área Bocaina I, as duas espécies de Ficus (F. glabra e F. insipida) atingiram 30,0\% do valor máximo que a variável valor de cobertura pode assumir, enquanto que $F$. insipida e Ficus cyclophyla atingiram $18,0 \%$ e $24,8 \%$ respectivamente nas áreas Bocaina II e Ilha Grande. Como a área basal é diretamente proporcional à biomassa, essas figueiras contribuem de modo muito significativo para a alocação da mesma nas formações secundárias estudadas.

Finalmente cabe ressaltar que a tendência de exemplares de Ficus spp. em atingir valores elevados de cobertura pode também ser detectado em outros estudos feitos em áreas de Mata Atlântica (Tab. 8). É relevante destacar que os trabalhos nela listados, feitos com metodologias distintas, foram feitos com desenhos amostrais que não tiveram como foco as figueiras, como no caso do presente estudo. 
Tabela 8. Valores de cobertura (VC) atingidos por exemplares de figueiras em diferentes estudos feitos na Mata Atlântica (ranking em relação ao número de espécies encontradas).

\begin{tabular}{|c|c|c|c|}
\hline espécie & autor & local & posição no VC \\
\hline F. insipida & este estudo & Mambucaba, RJ & 10 em 32 \\
\hline Ficus glabra & este estudo & Mambucaba, RJ & 10 em 32 \\
\hline F. ciclophylla & este estudo & Ilha Grande, RJ & 10 em 63 \\
\hline F. organensis & Silva 1989 & Morretes, PR & $10 \mathrm{em} 70$ \\
\hline F. gomelleira & Guedes-Bruni 1998 & Paraíso, RJ & 30 em 130 \\
\hline F. gomelleira & Freitas 2003 & Grumarí, RJ & 30 em 26 \\
\hline F. insipida & Solórzano 2006 & Camorim, RJ & 30 em 92 \\
\hline F. gomelleira & Stuzman et al. 2002 & Pariquera, SP & $6^{\circ}$ em 112 \\
\hline F. guaranitica & Cardoso-Leite et al. 2004 & Rio Claro, SP & $7^{\circ}$ em 39 \\
\hline F. obtusiuscula & Vilella et al. 1999 & Conquista, MG & 70 em 204 \\
\hline F. tomentella & Vilella et al. 1999 & Conquista, MG & $8^{\circ}$ em 204 \\
\hline F. gomelleira & Sambuichi 2006 & Ilhéus, BA & $8^{\circ}$ em 41 \\
\hline F. clusiaefolia & Kurtz \& Araújo 2000 & Guapimirim, RJ & $11^{\circ}$ em 138 \\
\hline F. insipida & Rodrigues \& Galvão 2006 & Paranapanema, SP & $12^{\circ}$ em 49 \\
\hline F. obtusiuscula & Toniato et al. 1998 & Campinas, SP & $12^{\circ}$ em 55 \\
\hline F. pulchella & Kurtz \& Araújo 2000 & Guapimirim, RJ & $13^{\circ}$ em 138 \\
\hline F. guaranitica & Silva \& Soares 2002 & São Carlos, SP & $21^{\circ}$ em 78 \\
\hline
\end{tabular}

Como em grande parte dos trabalhos acima foram feitos em formações secundárias, é muito possível que a posição destacada em relação ao valor de cobertura atingido por essas figueiras também possa ser atribuída ao mesmo tabu cultural, espalhado por extensas regiões do país. Este arquétipo, presente em várias culturas, trouxe como resultante cultural a preservação do abate de seus exemplares por parte das populações tradicionais brasileiras. Seja por um ou outro motivo cultural, as figueiras são muito frequentemente poupadas da derrubada quando as roças de subsistência são implantadas. Daí a constante presença destes exemplares de elevado porte, servindo como testemunho da historia local. Assim, considerando-se que alguns destes exemplares apresentam diâmetro e altura significativamente fora dos padrões das árvores que os circundam, isto leva a crer que em um dado momento da história, as áreas em estudo sofreram desmatamento ou algum tipo de exploração humana, porém, os exemplares de Ficus foram preservados. Dada a amplitude geográfica da situação de ocorrência dessas figueiras centenárias, é razoável se pensar que a decisão de preservação das mesmas esteja baseada em um mesmo tabu compartilhado por diferentes populações tradicionais (caiçaras, quilombolas, sitiantes, etc.).

História Ambiental e os aspectos biogeográficos de um tabuleiro 
A permanência destes exemplares de figueiras ao longo dos ciclos uso da paisagem pelas populações passadas redunda em resultantes ecológicas e biogeográficas. Primeiramente há que se destacar que, segundo Guedes-Bruni et al. (2009), várias espécies do gênero Ficus (como F. clusiaefolia Schott, F. gomelleira Kunth \& Bouché, F. insipida Willd. e F. organensis (Miq.) Miq.) destacam-se na paisagem florestal do Estado do Rio de Janeiro pelo atributo de raridade - isto é, ocorrem com a densidade de um indvíduo por hectare. De fato não foram encontrados exemplares em estágio não adulto nas áreas estudadas, o que leva a crer que o recrutamento da espécie esteja se dando de forma pouco intensa, pelo menos na escala espacial observada. Por outro lado, Guevara et al. (1986) apontam que árvores remanescentes são importantes para a sucessão ecológica, na medida em que provêem proteção e alimento para animais de fragmentos próximos. Estes autores ainda destacam o papel da avifauna na dispersão de frutos sob a copa destas árvores remanescentes. Ao longo dos trabalhos de campo e em contatos com moradores locais ficou evidenciado que estas figueiras representam um recurso significativo para a fauna local, tendo sido avistados o papagaio chauá (Amazona rodocorytha), o tucano (Ramphastos vitellinus ariel), araçarí (Selenidera maculirostris), o macaco-bugio (Alouatta fusca) e outros. Possivelmente um contingente muito maior de animais, especialmente os de hábito noturno, também faz uso desse recurso. A presença dessas árvores constitui, portanto, um importante elemento de atratibilidade da fauna dispersora de sementes. Pelo fato da estratégia de frutificação das espécies de Ficus se dar em grande abundância e por longo período, elas constituem uma espécie-chave para o ecossistema, no sentido empregado por Paine (1969). De fato, vestígios encontrados em algumas das figueiras estudadas (como jiraus e presença de alimentos usados para cevar a caça) levam a crer que as figueiras são espécies atrativas de caça, sendo usadas por caçadores como "árvore de espera" (Figura 3).

* Email: ritasvorc@gmail.com

** Departamento de Geografia, Centro de Ciências Sociais, Pontifícia Universidade Católica do Rio de Janeiro. Email: rro@puc-rio.br 

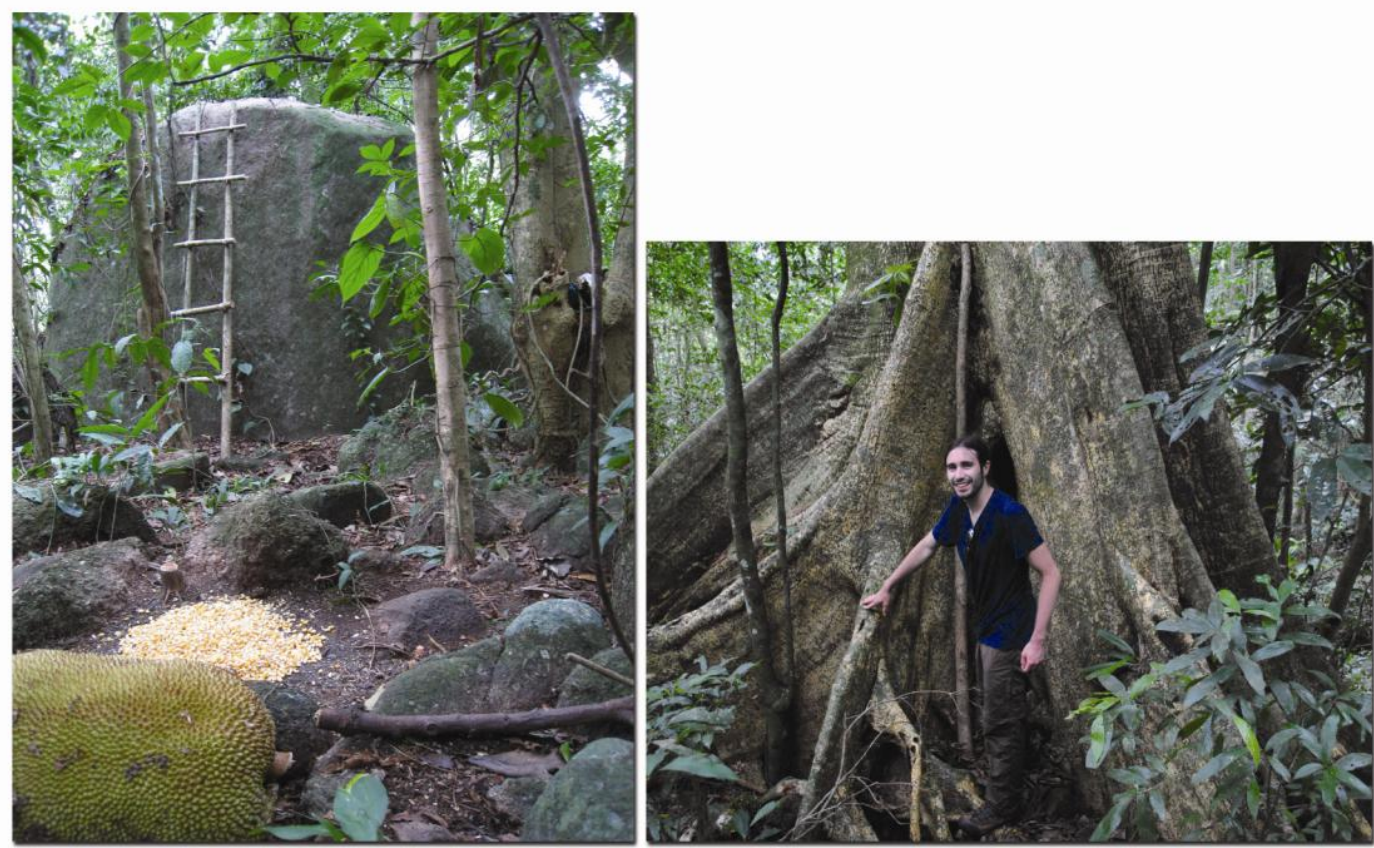

Uma ceva de caça clandestina, constituída por milho e jaca e o respectivo jirau, encontrados ao lado de um exemplar de Ficus ciclophylla (Ilha Grande, RJ).

Um conceito relevante na questão da preservação das figueiras e sua relação com a floresta circundante é o da chamada espécie-chave cultural, uma metáfora do conceito ecológico de espécie-chave. Estas constituem espécies "cujo impacto na sua comunidade ou ecossistema é grande e de forma desproporcional em relação à sua abundância" (POWER et al., 1996). Já Garibaldi \& Turner (2004) definem as espécies-chave culturais como aquelas que espelham a identidade cultural de um povo e que apresentam papéis fundamentais na dieta alimentar, no fornecimento de materiais, medicamentos ou voltadas para práticas espirituais. Estes papéis se desenvolvem ao longo do tempo histórico da relação entre populações e os ecossistemas e produzem importantes marcas na ligação entre sistemas ecológicos e sociais. Dentre estas marcas, os solos são o componente do ecossistema com a "memória" mais longa de distúrbios, mas a flora igualmente reflete por longo tempo os impactos humanos (BÜRGI \& GIMMI, 2007). Pode-se considerar ainda que estas figueiras demarquem as chamadas florestas culturais, no sentido empregado por Furlan (2006). Tratam-se de florestas manejadas pelas populações rurais, particularmente em áreas indígenas, comunidades ribeirinhas, seringueiros, quilombolas, caiçaras onde as comunidades tradicionais não têm a propriedade da terra e a ocupam e usam seus recursos de forma compartilhada.

As figueiras centenárias da Mata Atlântica constituem portanto verdadeiros marcos culturais. Tal fato evoca o aspecto simbólico da paisagem, ao expressar crenças, valores e mitos de uma população. Esta dimensão imaterial do paleoterritório, que se traduz em 
resultantes ecológicas concretas, traz à tona o largo espectro de ações de manejo dos ecossistemas por parte de populações tradicionais. A imponente presença dessas figueiras na Mata Atlântica contribui, de maneira clara, para opor "a idéia de natureza como externalidade ao humano" (REHBEIN e ROSS, 2010). E ainda, evidencia uma noção de ambiente que não se confunde com impactos na natureza, mas que privilegia as derivações e transformações destes lugares, a partir da construção da vida em sociedade com a natureza (SUETERGARAY, 2001). Ou seja, a transmutação do ecossistema em paisagem.

Alguns aspectos ecológicos e culturais acerca da presença destes exemplares podem ser ainda destacados a guisa de conclusão:

1) Como visto, os referidos exemplares do gênero Ficus encontrados nas áreas de estudos são distintos dos demais componentes arbóreos das áreas inventariadas no que se refere à sua biomassa. Particularmente os diâmetros e, em menor escala as alturas das figueiras, são significativamente mais elevados. Isto evidencia que se tratam de exemplares mais antigos do que as demais árvores que se encontram na mesma comunidade. Ou seja, em geral pertencem a outra geração, pois é evidente que há uma desproporção de diâmetros/alturas entre as figueiras e os demais componentes arbóreos. A presença das figueiras centenárias contribui para um aumento significativo da biomassa florestal na escala examinada.

2) Se as diferentes áreas geográficas do presente estudo (Serra da Bocaina e Ilha Grande) forem comparadas sob a influência de um mesmo marco cultural (as figueiras centenárias), observa-se que, apesar de distintos históricos de ocupação, a preservação destes exemplares se assemelha e sugere que o mesmo tabu encontra-se espalhado por distintas populações tradicionais do sudeste brasileiro.

3) Os enfoques da História Ambiental e da Biogeografia contribuem significativamente para ampliar a compreensão de processos ecológicos em territórios de populações tradicionais ao incorporar a dimensão cultural ao estudo da paisagem.

\section{Agradecimentos}

O presente trabalho é parte da dissertação de mestrado da primeira autora no Programa de Pós-Graduação em Ciências Ambientais e Florestais da UFRRJ. Rogério Oliveira é bolsista de pesquisa do Conselho Nacional de Desenvolvimento Científico e Tecnológico (CNPq). Os autores também são gratos pelo apoio recebido da curadora do Herbário do Departamento de Botânica da UFRRJ, à época Maria Verônica L. Pereira-Moura e do técnico de herbário Thiago de Azevedo Amorim. Agradecem também aos botânicos da UFRuraL Denise Monte Braz, Genise Vieira Somner, 
Helena Regina Pinto Lima, Inês Machline Silva, Joecildo Francisco Rocha, Lana da Silva Sylvestre, Maria Mercedes Teixeira da Rosa, Marilena Menezes Silva Conde, Pedro Germano Filho e aos do JBRJ, Ariane Luna Peixoto, Adriana Lobão, Elsie Franklin Guimarães, Marcelo de Souza, Haroldo Cavalcante de Lima, Marli Pires Morim e Sebastião José da Silva Neto pelo auxílio na determinação do material.

\section{REFERÊNCIAS BIBLIOGRÁFIAS}

ACOT, Pascal. História da ecologia. Rio de Janeiro: Campus, 1990.

ADAMS, Cristina. As populações caiçaras e o mito do bom selvagem: a necessidade de uma nova abordagem interdisciplinar. Revista de Antropologia v. 43, p. 145-182, 2000.

APG II (the Angiosperm Phylogeny Group). An update of the Angiosperm Phylogeny Group classification for the orders and families of flowering plants: APG II. Botanical Journal of the Linnean Society v.141, n. 4, p. :399-436, 2003.

BÜRGI, Matthias \& GIMMI, Urs. Three objectives of historical ecology: the case of litter collecting in Central European forests. Landscape Ecology v. 22, p. 7787, 2007.

CARAUTA, Jorge Pedro Pereira \& Diaz, Ernani. Figueiras do Brasil. Rio de Janeiro: Editora UFRJ/FAPERJ, 2003.

CARAUTA, Jorge Pedro Pereira. Ficus (Moraceae) no Brasil: Conservação e taxonomia. Albertoa, v. 2, p. 1-365, 1989.

CARDOSO-LEITE, Eliane.; COVRE, Tiago Batista, OMETO, Raquel Gardenal; CAVALCANTI, Denise Cidade \& PAGANI, Maria Iinez Fitossociologia e caracterização sucessional de um fragmento de mata ciliar, em Rio Claro/SP, como subsídio à recuperação da área. Revista do Instituto Florestal, v. 16, n. 1, p. : 31-41, 2004.

CRUMLEY C.L. (ed) Historical ecology: cultural knowledge and changing landscapes. Santa Fe: School of American Research Press, 1994.

DELAMONICA, Patricia.; LIMA, Denise. Flores.; OLIVEIRA, Rogério Ribeiro; MANTOVANI, Waldir. Estrutura e funcionalidade de populações de Miconia cinnamomifolia (DC.) Naud. em florestas secundárias estabelecidas sobre antigas roças caiçaras. Pesquisas Botânica, v.52, p. $125-142,2002$.

FONSECA, Denise Pini Rosalém. A marca do sagrado. Pp. 12-35. In: OLIVEIRA, Rogério Ribeiro de (ed). As marcas do Homem na floresta: História Ambiental de um trecho urbano de Mata Atlântica. Rio de Janeiro, Ed. PUC-Rio, 2005.

FREITAS, Marcelo Motta. Funcionalidade hidrológica dos cultivos de banana e territorialidades na paisagem do Parque Municipal de Grumari - Maciço da Pedra Branca-RJ. 2003. 150 f. Tese (Doutorado em Geografia) - Instituto de Geociências, Universidade Federal do Rio de Janeiro, Rio de Janeiro, 2003.

* Email: ritasvorc@gmail.com

** Departamento de Geografia, Centro de Ciências Sociais, Pontifícia Universidade Católica do Rio de Janeiro. Email: rro@puc-rio.br 
FURLAN, Sueli Ângelo. Florestas culturais: manejo sociocultural, territorialidades e sustentabilidade. Agrária, n. 3, p. 3-15, 2006.

GARCÍA-MONTIEL, Diana. El legado de la actividad humana en los bosques neotropicales contemporáneos. In: GUARIGAUTA, Manuel \& KATTAN, Gustavo (ed.). Ecologia y conservación de bosques neotropicales. Cartago: Ediciones LUR, p. 97-112, 2002.

GARIBALDI, Ann \& TURNER, Nancy 2004. Cultural keystone species: implications for ecological conservation and restoration. Ecology and Society, v. 9, n. 3). Disponível em http://www.ecologyandsociety.org/vol9/is s3/art1 (acesso em 034/02/2010).

GUEDES-BRUNI, Rejan Rodrigues. Composição, estrutura e similaridade florística de dossel em seis unidades fisionômicas de Mata Atlântica no Rio de Janeiro. 204 f. Tese (Doutorado em Ecologia). Instituto de Biociências, Universidade de São Paulo, São Paulo, 1998.

GUEDES-BRUNI, Rejan Rodrigues, SILVA, Ary Silva \& MANTOVANI, Waldir Rare canopy species in communities within the Atlantic Coastal Forest in Rio de Janeiro State, Brazil. Biodiversity and Conservation, v. 18, p.:387-403, 2009.

GUEVARA, Sergio; PURATA, Silvia E. \& van der MAAREL, Eddy. The role of remnant forest trees in tropical secondary succession. Vegetatio, v. 66, p.:77-84, 1986.

GUIMARÃES, Antony Érico. et al. Ecologia de mosquitos (Diptera: Culicidae) em áreas do Parque Nacional da Serra da Bocaina, Brasil.I - Distribuição por habitat. Revista de Saúde Pública, v. 34, n. 3, p.: 243-250, 2000.

HARTSHORN, Gary. Neotropical forest dynamics. Biotropica, v.12, p.:23-30, 1980.

KURTZ, Bruno Coutinho \& ARAÚJO, Dorothy Sue Dunn de. Composição florística e estrutura do componente arbóreo de um trecho de Mata Atlântica na Estação Ecológica do Paraíso, Cachoeiras do Macacú, RJ, Brasil. Rodriguesia v. 51, n. 78, p.: 69-112, 2000.
MUELLER-DOMBOIS, Dieter.; ELLENBERG, Heinz Aims and methods of vegetation ecology. New York, Willey \& Sons, 1974.

NAVEH, Z. What is holistic landscape ecology? A conceptual introduction. Landsccape and Urban Planning, v. 50, p. 7-26, 2000.

OLIVEIRA, Rogério Ribeiro de. Ação antrópica e resultantes a estrutura e composição da Mata Atlântica na Ilha Grande, RJ. Rodriguesia, v.53, n. 82, p.: 33-58, 2002.

OLIVEIRA, Rogério Ribeiro de. Environmental History, Traditional Populations, and Paleo-territoires in the Brazilian Atlantic Coastal Forest. Global Environment, v. I, p. 176-191, 2008.

PAINE, Richard. A note on trophic complexity and community stability. The American Naturalist, v.103, p.: 91-93, 1969.

POWER, Mary. Challenges in the quest for keystones. Bioscience, v. 46, n. 8, p. :609-620, 1996.

REHBEIN, Moisés Ortemar e ROSS, Jurandyr Luciano Sanches. Ambiente; urbano; impacto - impacto ambiental urbano: revisões e construções de significados. GEOUSP - Espaço e Tempo, São Paulo, No 27, pp. 95 - 112, 2010.

RODRIGUES, Elisângela Ronconi \& GALVÃO, Franklin. Florística e fitossociologia de uma área de reserva legal recuperada por meio de sistema agroflorestal na região do Pontal do Paranapanema, São Paulo. Floresta. v. 36, n. 2, p.: 295-3003, 2006.

SAMBUICHI, Regina Helena Rosa. Structure and dynamics of the tree community in a "cabruca" area in the cacao region of southern Bahia State, Brazil. Acta Botanica Brasilica, v. 20, n. 4, p. : 943-954, 2006.

SANTOS, M. A natureza do espaço. São Paulo: EDUSP. 2006

SILVA, Fernando Cardoso. Composição florística e estrutura fitossociológica da Floresta Tropical Ombrófila da encosta atlântica no município de Morretes (Paraná). Boletim de Pesquisa Florestal , v. 18/19, p.: 31-49, 1989. 
SILVA, Luciana Álvares \& SOARES, João Juares. Levantamento fitossociológico em um fragmento de floresta estacional semidecídua, no município de São Carlos, SP. Acta Botanica Brasilica, v. 16, n. 2, p.: 205-216, 2002.

SIMMONS, Ian. Changing the face of the earth: culture, environment, history. Cambridge, Balackwell Publishers Inc., 1996.

SOLLINS, Phillip. Factors influencing species composition in tropical lowland rain forest: does soil matter? Ecology, v. 79, n. 1, p. :23-30, 1998.

SOLÓRZANO, Alexandro. Composição florística, estrutura e história ambiental em áreas de Mata Atlântica no Parque Estadual da Pedra Branca, Rio de Janeiro, RJ. 151 f. Dissertação (Mestrado em Botânica). Instituto de Pesquisas Jardim Botânico do Rio de Janeiro/Escola Nacional de Botânica Tropical, Rio de Janeiro, 2006.

SOUZA, Marcos Barros de \& MARIANO, Zilda de Fátima. Geografia Física e a questão ambiental no Brasil. GEOUSP Espaço e Tempo, n. 23, p. 77 - 98, 2008.

SUETERGARAY, Dirce Maria Antunes. Espaço geográfico uno e múltiplo. Scripta Nova, n. 93, 2001

SWETNAM, Thomas; ALLEN, Craig \& BETANCOURT, Julio. Applied historical ecology: Using the past to manage the future. Ecological Applications, v. 9, n. 4, p. : 1189-1206, 1999.
SZTUTMAN, Marcio \& RODRIGUES, Ricardo Ribeiro. Vegetational mosaic of contiguous forest area in a coastal plain, Campina do Encantado State Park, Pariquera-Açu, SP. Revista Brasileira de Botânica, v. 25, n. 2, p. : 161-176, 2002.

TONIATO, Maria Teresa Zugliani; LEITÃO FILHO, Hermógenes de Freitas. \& RODRIGUES, R.R. Fitossociologia de um remanescente de floresta higrófila (mata de brejo) em Campinas, SP. 1998. Revista Brasileira de Botânica, v. 21, n. 2, p. : 197-210, 1998.

VERGER, Pierre. Ewé: o uso das plantas na sociedade iorubá. São Paulo: Companhia das Letras, 1995.

VILELA, E.A.; Oliveira Filho, A.T. \& Carvalho, D.A. Fitossociologia de floresta ripária do médio rio Grande, Conquista, MG. Revista Árvore, v. 23, p.: 423-433, 1999.

VUONO, Yara Struffaldi de. Inventário fitossociológico. In: SYLVESTRE, Lana da Silva \& ROSA, Maria Mercedes Teixeira da. Manual metodológico para estudos botânicos na Mata Atlântica. Seropédica: EDUR. 2002. p.: 51-65, 2002.

ZAR, J. H. Biostatistical analysis. 3. ed. New Jersey: Prentice-Hall International. 1996. 
